\title{
Composite TeXture Shape Classification BASED ON MORPHOLOGICAL SKELETON AND REGIONAL MOMENTS
}

\author{
M. Rama Bai \\ Department of Computer Engineering, M.G.I.T, Hyderabad, Andhra Pradesh, India \\ vallapu.rama@gmail.com
}

\begin{abstract}
After several decades of research, the development of an effective feature extraction method for texture classification is still an ongoing effort. Therefore, several techniques have been proposed to resolve such problems. In this paper a novel composite texture classification method based on innovative pre-processing techniques, skeletonization and Regional moments (RM) is proposed. This proposed texture classification approach, takes into account the ambiguity brought in by noise and the different caption and digitization processes. To offer better classification rate, innovative pre-processing methods are applied on various texture images first. Pre-processing mechanisms describe various methods of converting a grey level image into binary image with minimal consideration of the noise model. Then shape features are evaluated using RM on the proposed Morphological Skeleton (MS) method by suitable numerical characterization measures for a precise classification. This texture classification study using MS and RM has given a good performance. Good classification result is achieved from a single region moment RM10 while others failed in classification.
\end{abstract}

\section{KEYWORDS}

Classification, Pre-processing, Morphological Skeleton, Hu Moments, Regional Moments

\section{INTRODUCTION}

Texture classification is an important step in many computer vision algorithms [1,2,3,4]. In texture classification, images of same group should be homogeneous with respect to some characteristics or features and different textures should have significant different features or characteristics.

We know a good shape representation should provide an accurate and complete description of a given object. One of the great advantages of shape representation is, only shape can be preserved instead of the whole image. By this, the storage size will be reduced and the original image can be reconstructed from the preserved shape. A simple and compact representation of a shape that preserves many topological characteristics like size, length of a shape, separation of the shapes, and other qualitative behavioral aspects of the shape can be provided by a skeleton representation. Such kind of shape representation schemes is useful for fast image retrieval and in image compression problems. By preserving intrinsic details of shape the original image can be reconstructed in skeletonization approach. Thus it plays an essential function in human visual perception in shape recognition and shape analysis problems.

It was Cayley and Sylvester who initially derived the theory of moment invariants based on analytical geometry. Using their study it was Hu who first introduced the concept of algebraic 
Signal \& Image Processing : An International Journal (SIPIJ) Vol.4, No.3, June 2013

moment invariants. The set of Hu moments are invariant to any change in an object subjected to rotation, scale and translation change [5-9]. Most of the shape representation algorithms using moment invariants consider all pixels of a given image in classification taking a long time to compute and thus are relatively less inefficient. To overcome this deficiency, a novel method is proposed which extracts the skeleton of target image by applying preprocessing methods first, then Regional Moments (RM) derived from Hu moments are applied on the extracted skeleton to achieve classification in efficient manner.

The paper is organized as follows. Section 2 briefly described the methodology employed introducing preprocessing methods, skeletonization, $\mathrm{Hu}$ moments, regional moments and the classification algorithm, section 3 deals with results and discussions. Finally in section 4 conclusions are listed.

\section{METHODOLOGY}

The present section briefly outlines an effective method of shape classification by combining innovative pre-processing techniques, morphological skeletonization method and regional moments as shown in Figure 1. Pre-processing mechanism describes various methods of converting a grey level image into binary image. In this paper, six different pre-processing techniques are studied. They are mean, median, mode, maximum, minimum and max-min. The binary images obtained from those pre-processing techniques are then subjected to skeletonization using morphological methods. In other words, the most possible reduced image (skeleton) is obtained. Further, to the skeleton extracted binary images the paper evaluated ten regional moments on five groups of shape pattern images and derived an effective classifier.

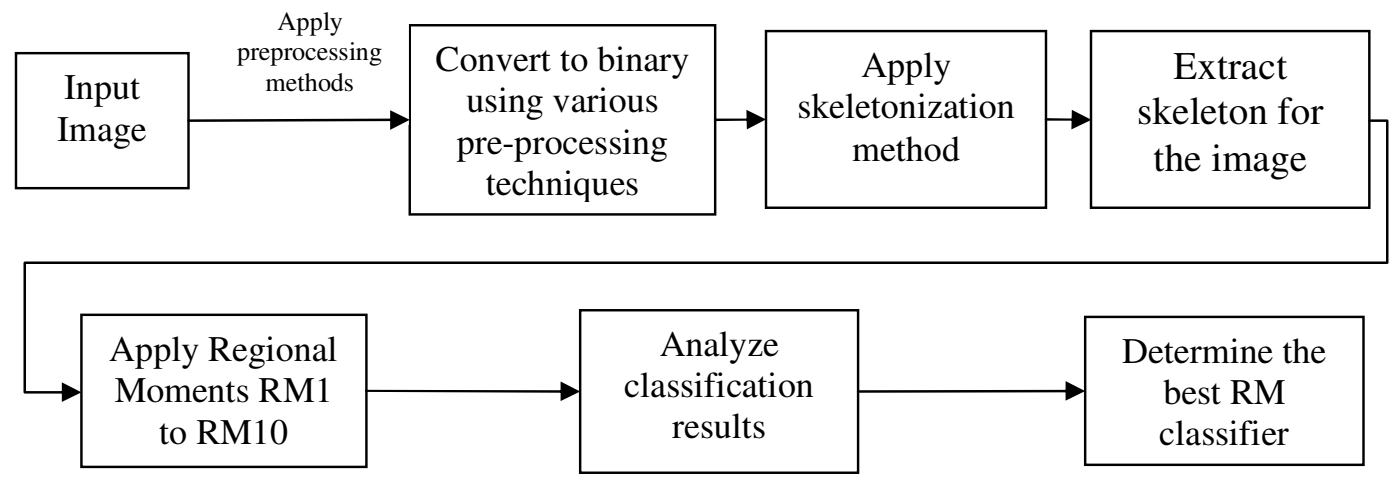

Figure 1. Proposed block diagram for Texture classification

\subsection{Skeleton and shape}

One key technique for shape representation is the skeletonization approach. It has been studied widely since skeletons have important properties which make them suitable for structural pattern recognition $[10,11]$. Skeletonization methods are of two types: pixel-based and non-pixel-based. In a pixel-based method, all pixels in a shape are utilized in the skeletonization process. Pixel based methods often use thinning techniques [11, 12] or distance transforms [13,12]. Contour pixel of a shape is used for non-pixel-based skeletonization method. Hence, logically one can say the skeleton shape is represented by its contour $[14,15]$.

For this, the present section proposes a novel approach for skeletonization based on MS approach. One of the disadvantages of the existing skeletonization method is that it is not automatic and needs human interaction. The novelty of the paper is that it advocated an innovative approach for 
Signal \& Image Processing : An International Journal (SIPIJ) Vol.4, No.3, June 2013

the extraction of skeleton based on MS scheme, which is completely based on morphology, for the classification purpose.

The MS scheme is a leading morphological shape representation algorithm [16, 26, 27]. In the MS scheme, a given shape is represented as a union of all maximal disks contained in the shape. The advantages of this basic algorithm include that they have simple and well-defined mathematical characterizations and they are easy and efficient to implement.

In MS scheme the skeleton of an image(I) is derived in terms of simple morphological operations erosions and openings. The morphological skeleton $S_{k}$ of an image is defined by the Equation 1.

$$
\begin{aligned}
& \mathrm{S}_{\mathrm{k}}(\mathrm{I})=(\mathrm{I} \theta \mathrm{kB})-(\mathrm{I} \theta \mathrm{kB})^{\circ} \mathrm{B} \\
& \text { Where } \mathrm{I}, \theta_{,-,} \circ, \mathrm{B} \text { are } \\
& \text { I Denotes texture images } \\
& \text { - Denotes subtraction } \\
& \theta \text { Denotes morphological erosion operation } \\
& \text { - Denotes morphological opening operation } \\
& \text { B Denotes structuring element. } \\
& \text { K Denotes successive erosions of I. }
\end{aligned}
$$

\subsection{Hu Moment Invariants}

An image classification problem involves sorting images based on their shape features. This is achieved by suitable characterization of the object shape. One can easily say whether a given image fall into the same set based on an important rule. That is ideally one can say dissimilar objects should have dissimilar categorization and similar objects should have similar description.

A group of algebraic moments based on the combination of general moments were proposed for the first time by $\mathrm{Hu}$ and were known as $\mathrm{Hu}$ moments. A greater part of image recognition experiments achieved good outcomes using those $\mathrm{Hu}$ moments[17-21]. These moments have achieved good results in the majority of $2 \mathrm{D}$ and $3 \mathrm{D}$ image recognition experiment. Let $\mathrm{X}$ and $\mathrm{Y}$ represent the horizontal and vertical axis in 2-Dimension, a point $(\mathrm{x}, \mathrm{y})$ represents the gray level value in the image and is given as $f(x, y)$.

The $(p+q)$ th two dimensional moment is defined as follows:

$\mathrm{m}_{\mathrm{pq}}=\int_{\mathrm{x}, \mathrm{y} \in \mathrm{c}} \int \mathrm{x}^{\mathrm{p}} \mathrm{y}^{\mathrm{q}} \mathrm{f}(\mathrm{x}, \mathrm{y}) \mathrm{dxdy} \quad \mathrm{p}, \mathrm{q}=0,1, \ldots \ldots$.

The $(p+q)$ th two dimensional central moment is defined as follows

$\mu_{\mathrm{pq}}=\int_{\mathrm{x}, \mathrm{y} \in \mathrm{c}} \int(\mathrm{x}-\overline{\mathrm{x}})^{\mathrm{p}}(\mathrm{y}-\overline{\mathrm{y}})^{\mathrm{q}} \mathrm{f}(\mathrm{x}, \mathrm{y}) \mathrm{dxdy}$

Where

$\bar{x}=\frac{m_{10}}{m_{00}}=\frac{\int_{x, y \in c} \int x f(x, y) d x d y}{\int_{x, y \in c} \int f(x, y) d x d y}$ 
$\bar{y}=\frac{m_{01}}{m_{00}}=\frac{\int_{x y \in c} \int y f(x, y) d x d y}{\int_{x, y \in c} \int f(x, y) d x d y}$

The $(p+q)$ th two dimensional normalized central moment is defined as follows

$\eta_{\mathrm{pq}}=\frac{\mu_{\mathrm{pq}}}{\frac{\mu_{00} \mathrm{pq}+2}{\mu_{00}}}$

The HMincludes invariants up to the third order. The HMs is given by HM1 to HM7 in equations 7 to 13 .

The seven Hu moments are:

$$
\begin{aligned}
\text { HM1 }= & \eta_{20}+\eta_{02} \\
\text { HM2 }= & \left(\eta_{20}-\eta_{02}\right)^{2}+4 \eta_{11}^{2} \\
\text { HM3 }= & \left(\eta_{30}+\eta_{12}\right)^{2}+\left(3 \eta_{21}-\eta_{03}\right)^{2} \\
\text { HM }= & \left(\eta_{30}+\eta_{12}\right)^{2}+\left(\eta_{21}+\eta_{03}\right)^{2} \\
\text { HM5 }= & \left(\eta_{30}+\eta_{12}\right)\left(\eta_{30}-3 \eta_{12}\right)\left[\left(\eta_{30}+\eta_{12}\right)^{2}-3\left(\eta_{21}+\eta_{03}\right)^{2}\right] \\
& +3\left(\eta_{21}+\eta_{03}\right)\left(\eta_{21}-\eta_{03}\right)\left[3\left(\eta_{30}+\eta_{12}\right)^{2}-\left(\eta_{21}+\eta_{03}\right)^{2}\right] \\
\text {HM}_{6}= & \left(\eta_{20}-\eta_{02}\right)\left[\left(\eta_{30}+\eta_{12}\right)^{2}-\left(\eta_{21}+\eta_{03}\right)^{2}\right]+4 \eta_{11}\left(\eta_{30}+\eta_{12}\right)\left(\eta_{21}+\eta_{03}\right) \\
\text { HM7 }= & \left(\eta_{30}+\eta_{12}\right)\left(3 \eta_{21}-\eta_{03}\right)\left[\left(\eta_{30}+\eta_{12}\right)^{2}-3\left(\eta_{21}+\eta_{03}\right)^{2}\right] \\
+ & \left(\eta_{21}+\eta_{03}\right)\left(3 \eta_{12}-\eta_{30}\right)\left[3\left(\eta_{30}+\eta_{12}\right)^{2}-\left(\eta_{21}+\eta_{03}\right)^{2}\right]
\end{aligned}
$$

\subsection{Regional Moments on MS}

To illustrate shape of an object or to match it with patterns of similar shape and for classification problems based on shapes, moment invariants have been in use for a long time. A variety of texture classification methods are proposed in the literature for the past three decades. But so far no one has attempted classification of textures based on MS schemes especially using RM. The shape parameter derived from objects help in attaining it. Hence, the present study derived RM based on HM.

The RMs is given by RM1 to RM10 in equations 14 to 23 .

$$
\begin{aligned}
& \mathrm{RM} 1=\frac{\sqrt{\mathrm{HM} 2}}{\mathrm{HM} 1} \\
& \mathrm{RM} 3=\frac{\sqrt{\mathrm{HM} 3}}{\sqrt{\mathrm{HM} 4}} \\
& \text { RM5 }=\sqrt{\frac{\text { HM4 }}{\sqrt{\mid \text { HMS }}}} \\
& \mathrm{RM} 7=\sqrt{\frac{|\mathrm{HM} 6|}{\mathrm{HM} 1 \times \sqrt{\mathrm{HMS}}}} \\
& \text { RM9 }=\sqrt{\frac{\mid \text { HM6 } \mid}{\sqrt{\text { HM2 } \times \mid \text { HM5 } \mid}}} \\
& \mathrm{RM} 2=\sqrt{\frac{\mathrm{HM} 1+\sqrt{\mathrm{HM} 2}}{\mathrm{HM} 1-\sqrt{\mathrm{HM} 2}}} \\
& \text { RM4 }=\frac{\text { HM3 }}{\sqrt{\mid \text { HMS }}} \\
& \text { RM6 }=\sqrt{\frac{|\mathrm{HM} 6|}{\mathrm{HM} 1 \times \mathrm{HM} 3}} \\
& \text { RM8 }=\sqrt{\frac{\mid \text { HM6 } \mid}{\text { HM1 } \times \text { HM4 }}} \\
& \text { (22) } \mathrm{RM} 10=\sqrt{\frac{|\mathrm{HM}|}{\mathrm{HM} 2 \times \mathrm{HM} 3}}
\end{aligned}
$$


Signal \& Image Processing : An International Journal (SIPIJ) Vol.4, No.3, June 2013

\subsection{Classification of shape texture images based on RM}

To demonstrate the classification process five sets of images with dissimilar shapes like brick, circle, curves, lines and zigzag are considered. Each set consists of ten images of similar shape. These texture images are displayed from Figure 2 to Figure 6. The classification process is given in Algorithm 1 below.
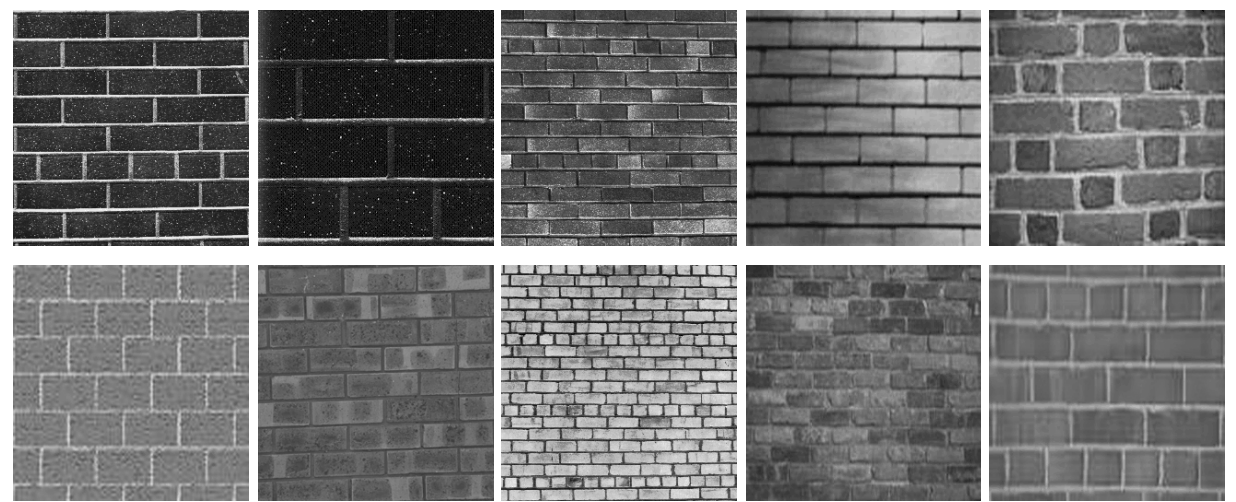

Figure 2. Original images of brick textures.
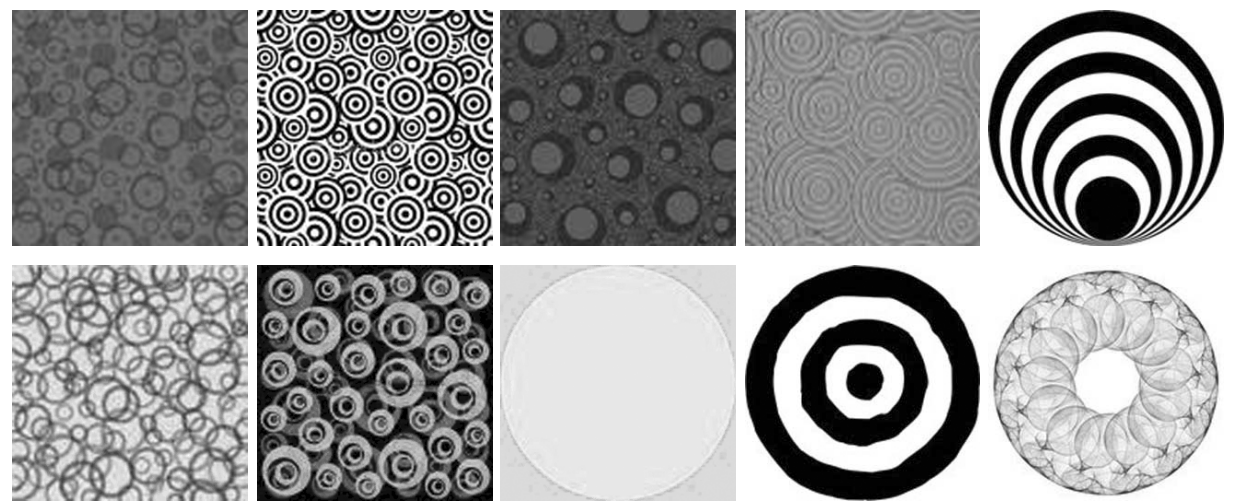

Figure 3. Original images of Circles textures.
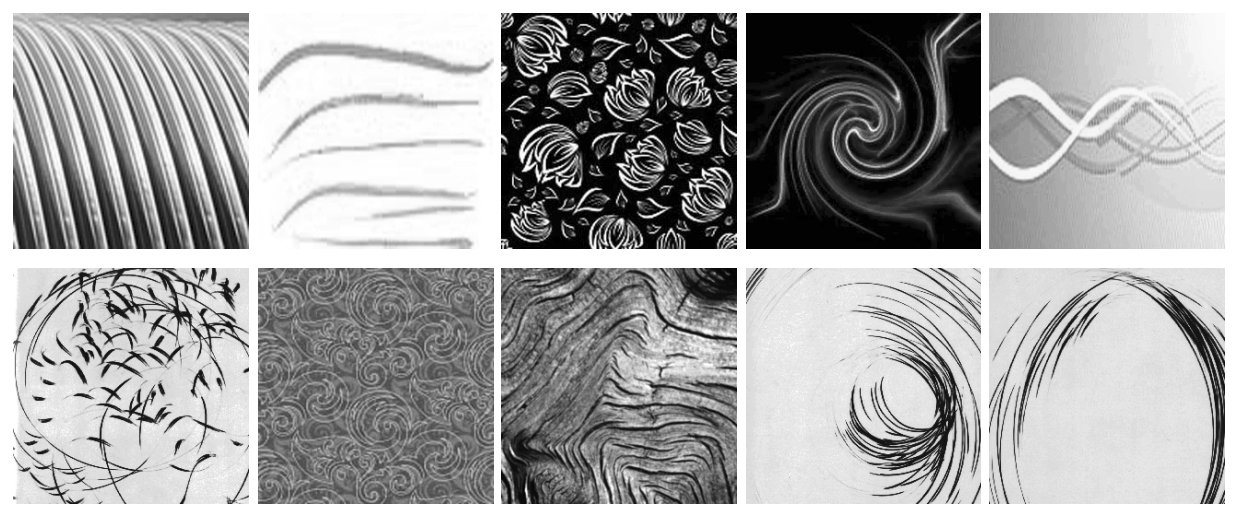

Figure 4. Original images of Curves textures. 
Signal \& Image Processing : An International Journal (SIPIJ) Vol.4, No.3, June 2013

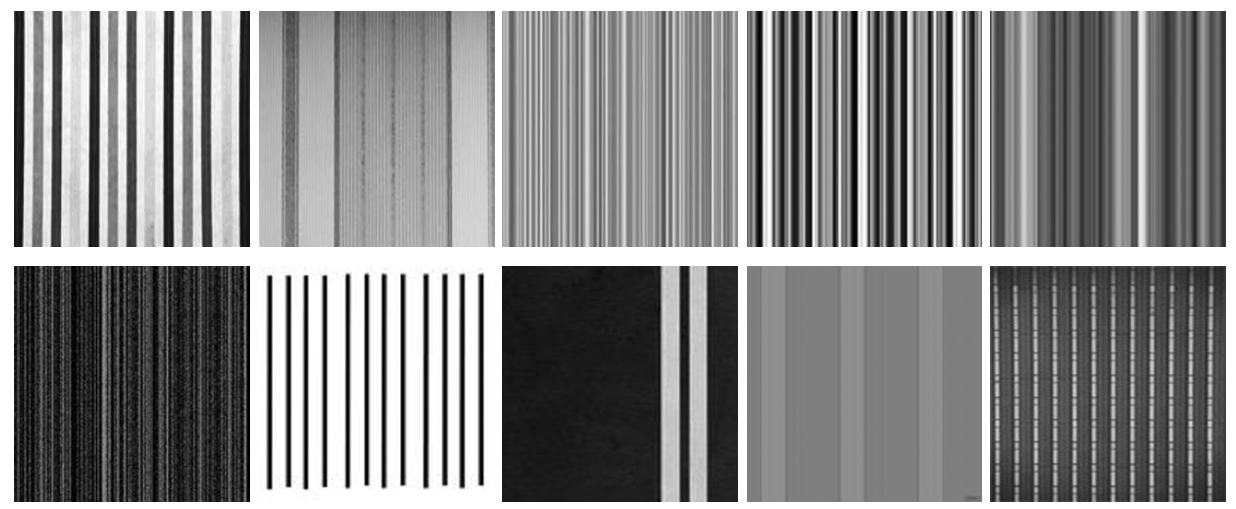

Figure 5. Original images of Line textures.

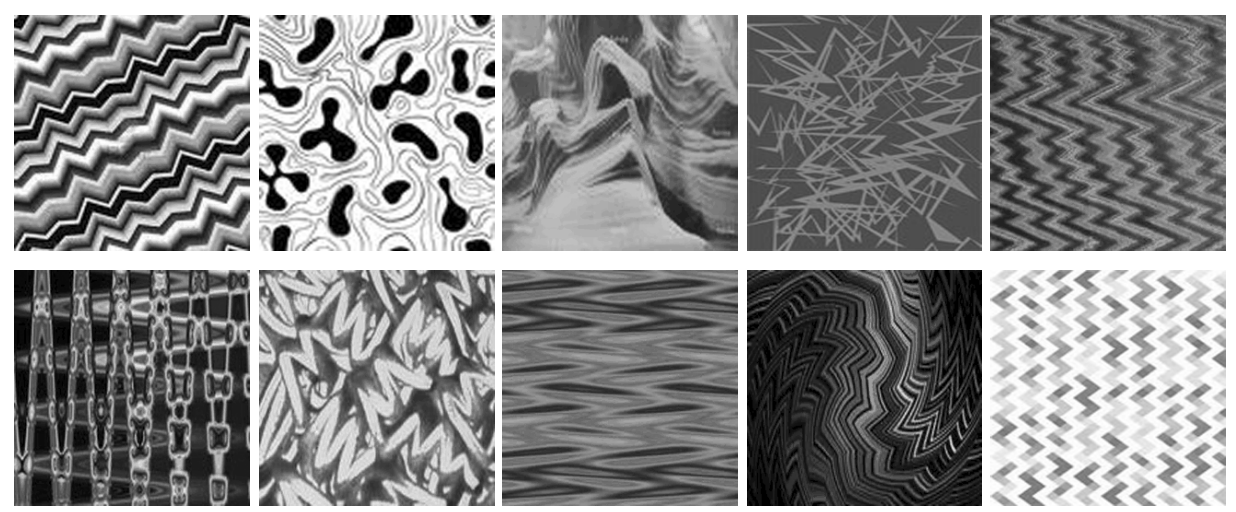

Figure 6. Original images of Zigzag textures.

\section{Algorithm 1: A Novel textures classification method using MS and RM}

Classification is performed on five groups of texture images by the following steps.

Step 1: Read the gray scale texture images and change it into a binary one.

Step 2: A binary images is obtained by applying six different pre-processing techniques like mean, median, mode, maximum, minimum and max-min.

Step 3: Each pre-processed binary image is then subjected to skeletonization using the MS scheme.

Step 4: Evaluate RM on the skeleton texture generated.

Step 5: On each set consisting of ten textures images, evaluate average values of each RM's and store them in a database.

Step 6: Plot the classification graph for all ten RM on MS scheme and determine the significant RM that classifies accurately and efficiently the given textures.

\section{RESULTS AND DISCUSSIONS}

\subsection{Classification on shape texture by $\mathrm{RM}$ using local maximum pre-processing and MS method}

In order to illustrate the classification problem five sets of different shape textures are taken. Each set comprises of ten images of similar shapes. For all set the average value of each regional 
Signal \& Image Processing : An International Journal (SIPIJ) Vol.4, No.3, June 2013

moment from 1 to 10 is computed and given in the Table 1. A graph is also displayed based on these values as shown in Figure 7.

Table 1. Average RM on MS schemes obtained after pre-processing the image using local maximum.

\begin{tabular}{|l|c|c|c|c|c|c|c|c|c|c|}
\hline Image & RM1 & RM2 & RM3 & RM4 & RM5 & RM6 & RM7 & RM8 & RM9 & RM10 \\
\hline Brick & 0.0898 & 1.1107 & 2.9135 & 2.3506 & 0.8937 & 0.1342 & 0.1860 & 0.2199 & 0.7803 & 59.2652 \\
\hline Circle & 0.0308 & 1.0313 & 2.1898 & 3.3016 & 1.2229 & 0.1342 & 0.1739 & 0.1374 & 1.4000 & 133.2895 \\
\hline Curve & 0.1234 & 1.1435 & 1.6063 & 1.7279 & 1.2570 & 0.3019 & 0.3401 & 0.2549 & 0.6254 & 83.0574 \\
\hline Line & 0.1639 & 1.4251 & 1.3655 & 1.5130 & 1.0728 & 0.2716 & 0.3398 & 0.3208 & 0.9978 & 115.2402 \\
\hline Zigzag & 0.0994 & 1.1127 & 2.3796 & 2.1447 & 1.0944 & 0.1921 & 0.2337 & 0.2120 & 0.8345 & 38.7425 \\
\hline
\end{tabular}

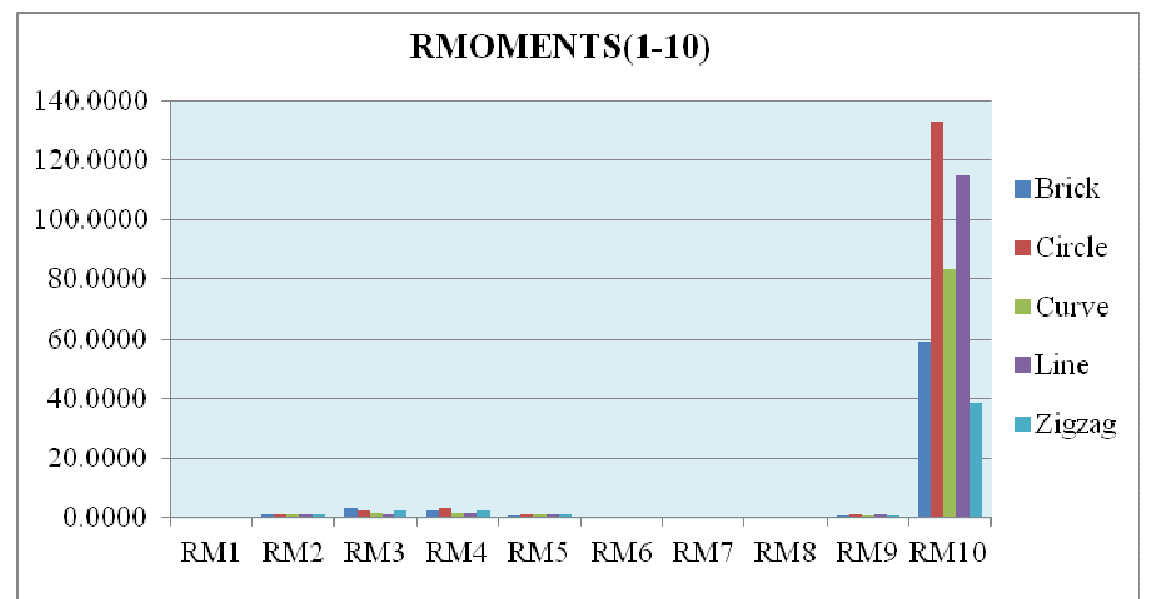

Figure 7. Classification graph on average RM's obtained after pre-processing texture images using local maximum

\subsection{Classification on shape texture by RM using local minimum pre-processing and MS method}

The Algorithm 1 is applied on the same texture images after applying local minimum preprocessing method. The computed values are given in Table 2. A graph is also displayed based on these values. Figure 8 gives a representation of it.

Table 2. Average RM on MS schemes obtained after preprocessing the image using local minimum.

\begin{tabular}{|c|c|c|c|c|c|c|c|c|c|c|}
\hline Image & RM1 & RM2 & RM3 & RM4 & RM5 & RM6 & RM7 & RM8 & RM9 & RM10 \\
\hline Brick & 0.0756 & 1.0831 & 2.1575 & 1.9674 & 1.0595 & 0.1143 & 0.1863 & 0.1780 & 0.7368 & 56.1803 \\
\hline Circle & 0.0436 & 1.0449 & 3.1076 & 2.2917 & 1.0493 & 0.1069 & 0.1400 & 0.1419 & 0.8891 & 47.2131 \\
\hline Curve & 0.1746 & 1.2078 & 1.3197 & 1.4866 & 1.2078 & 0.2758 & 0.3816 & 0.2927 & 0.8514 & 43.0058 \\
\hline Line & 0.1720 & 1.4639 & 1.2169 & 1.2872 & 1.0428 & 0.2842 & 0.3268 & 0.3241 & 0.9273 & 66.6750 \\
\hline Zigzag & 0.1115 & 1.1221 & 2.3051 & 2.0425 & 1.0191 & 0.1370 & 0.2164 & 0.2183 & 0.6487 & 32.5595 \\
\hline
\end{tabular}


Signal \& Image Processing : An International Journal (SIPIJ) Vol.4, No.3, June 2013

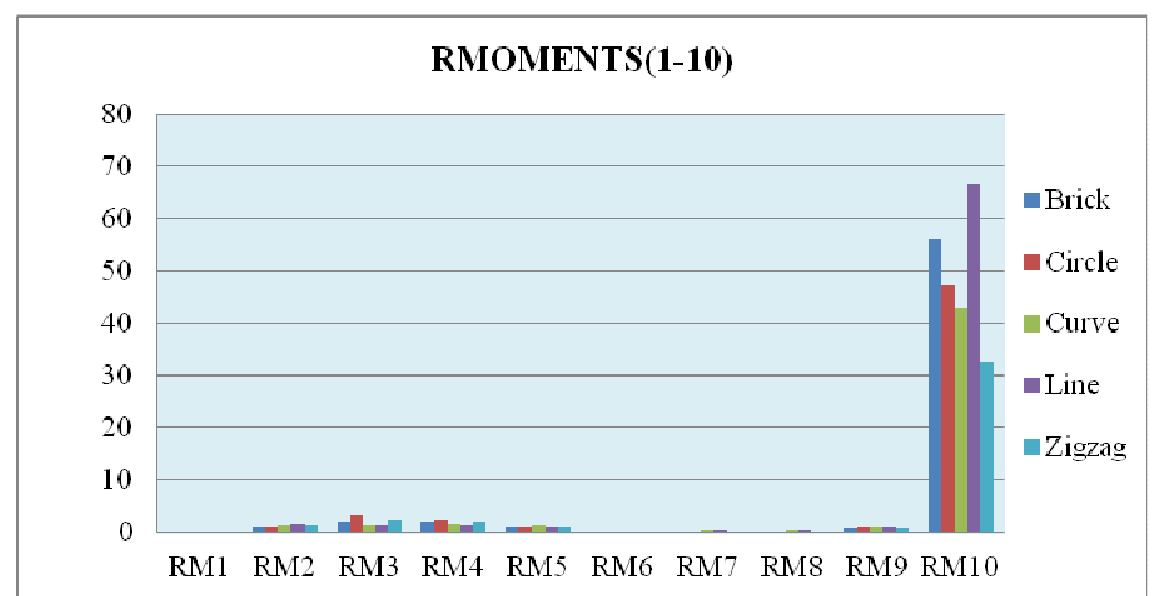

Figure 8. Classification graph on average RM's obtained after pre-processing texture images using local minimum

\subsection{Classification on shape texture by RM using local max-min pre-processing and MS method}

The Algorithm 1 is applied on the same texture images after applying local Max-Min preprocessing method. The computed values are given in Table 3. A graph is also displayed based on these values. Figure 9 gives a representation of it.

Table 3. Average RM on MS schemes obtained after pre-processing the image using local maxmin.

\begin{tabular}{|c|c|c|c|c|c|c|c|c|c|c|}
\hline Image & RM1 & RM2 & RM3 & RM4 & RM5 & RM6 & RM7 & RM8 & RM9 & RM10 \\
\hline Brick & 0.0333 & 1.0340 & 2.0578 & 1.7756 & 1.3190 & 0.3052 & 0.1750 & 0.1297 & 0.8208 & 96.4573 \\
\hline Circle & 0.0194 & 1.0198 & 1.4130 & 1.5529 & 1.2337 & 0.1710 & 0.1210 & 0.1023 & 0.9466 & 269.5946 \\
\hline Curve & 0.1376 & 1.1544 & 4.8250 & 4.1798 & 1.0732 & 0.3138 & 0.3206 & 0.2890 & 0.9263 & 69.0026 \\
\hline Line & 0.0689 & 1.0740 & 1.1491 & 1.1258 & 1.1157 & 0.3371 & 0.2675 & 0.2274 & 1.0667 & 231.0970 \\
\hline Zigzag & 0.0792 & 1.0876 & 1.7605 & 1.7629 & 1.1150 & 0.1125 & 0.1780 & 0.1591 & 0.5890 & 89.6390 \\
\hline
\end{tabular}

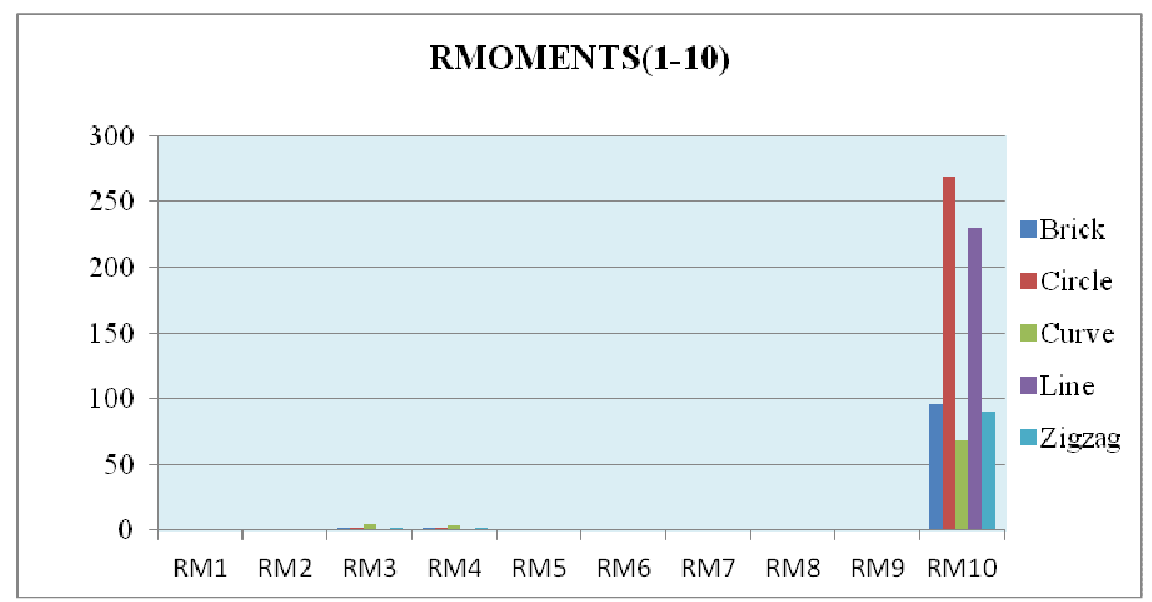

Figure 9. Classification graph on average RM's obtained after pre-processing texture images using local max-min 
Signal \& Image Processing : An International Journal (SIPIJ) Vol.4, No.3, June 2013

\subsection{Classification on shape texture by RM using local mode pre-processing and MS method}

The Algorithm 1 is applied on the same texture images after applying local mode pre-processing method. The computed values are given in Table 4. A graph is also displayed based on these values. Figure 10 gives a representation of it.

Table 4. Average RM on MS schemes obtained after pre-processing the image using local mode

\begin{tabular}{|l|l|l|l|l|l|l|l|l|l|l|}
\hline Image & RM1 & RM2 & RM3 & RM4 & RM5 & RM6 & RM7 & RM8 & RM9 & RM10 \\
\hline Brick & 0.0754 & 1.0871 & 2.1417 & 1.7884 & 1.0626 & 0.1429 & 0.1811 & 0.1787 & 0.9167 & 86.4546 \\
\hline Circle & 0.0276 & 1.0283 & 2.1994 & 2.0984 & 1.0566 & 0.0756 & 0.1259 & 0.1176 & 0.9296 & 1299.9533 \\
\hline Curve & 0.1640 & 1.1880 & 1.2157 & 1.3007 & 1.1373 & 0.3213 & 0.3586 & 0.3181 & 0.9417 & 72.5362 \\
\hline Line & 0.1443 & 1.4063 & 1.5700 & 1.5445 & 1.2058 & 0.3532 & 0.3068 & 0.2668 & 1.0231 & 74.9628 \\
\hline Zigzag & 0.0849 & 1.0917 & 1.7182 & 1.8181 & 1.2964 & 0.1815 & 0.2437 & 0.1944 & 0.9972 & 43.5181 \\
\hline
\end{tabular}

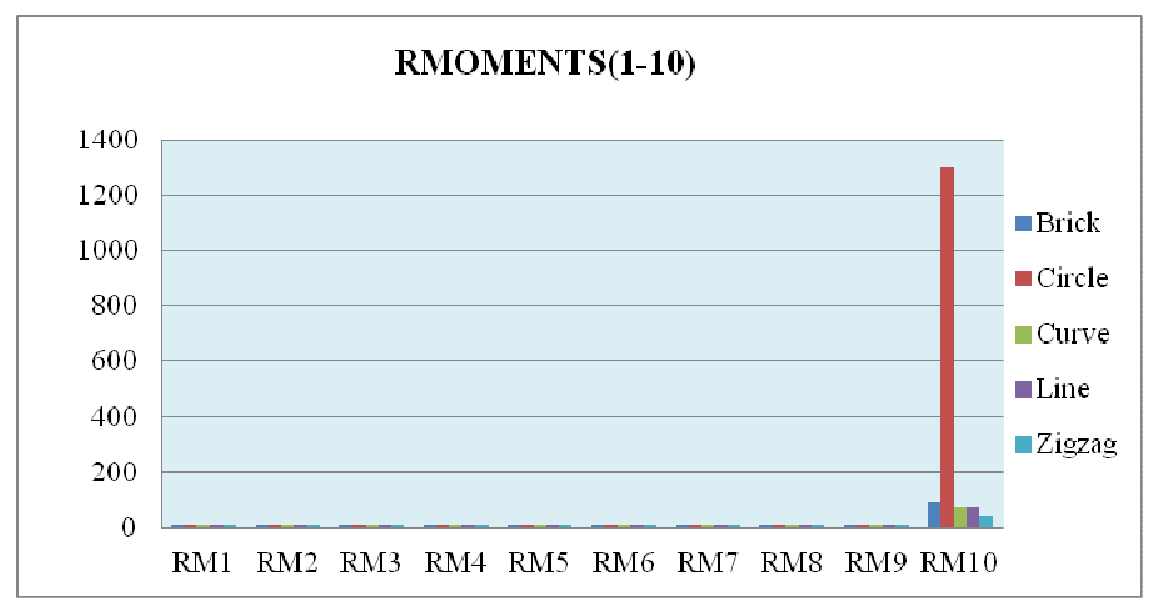

Figure 10. Classification graph on average RM's obtained after pre-processing texture images using local mode

\subsection{Classification on shape texture by RM using local mean pre-processing and MS method}

The Algorithm 1 is applied on the same texture images after applying local mean pre-processing method. The computed values are given in Table 5. A graph is also displayed based on these values. Figure 11 gives a representation of it.

Table 5. Average RM on MS schemes obtained after preprocessing the image using local mean

\begin{tabular}{|c|c|c|c|c|c|c|c|c|c|c|}
\hline Image & RM1 & RM2 & RM3 & RM4 & RM5 & RM6 & RM7 & RM8 & RM9 & RM10 \\
\hline Brick & 0.0815 & 1.0960 & 2.4534 & 2.4520 & 1.1317 & 0.1576 & 0.2333 & 0.2083 & 1.1167 & 102.0747 \\
\hline Circle & 0.0297 & 1.0303 & 3.8515 & 2.6614 & 0.8926 & 0.0761 & 0.1101 & 0.1294 & 0.6858 & 249.6272 \\
\hline Curve & 0.1705 & 1.2024 & 2.8460 & 2.2374 & 1.1046 & 0.2557 & 0.3830 & 0.3262 & 0.8872 & 40.6571 \\
\hline Line & 0.1637 & 1.4257 & 1.2451 & 1.2122 & 1.1184 & 0.3930 & 0.3555 & 0.3151 & 1.0295 & 118.1763 \\
\hline Zigzag & 0.1087 & 1.1224 & 1.6402 & 1.5388 & 1.0120 & 0.1886 & 0.2285 & 0.2254 & 0.6376 & 40.9032 \\
\hline
\end{tabular}


Signal \& Image Processing : An International Journal (SIPIJ) Vol.4, No.3, June 2013

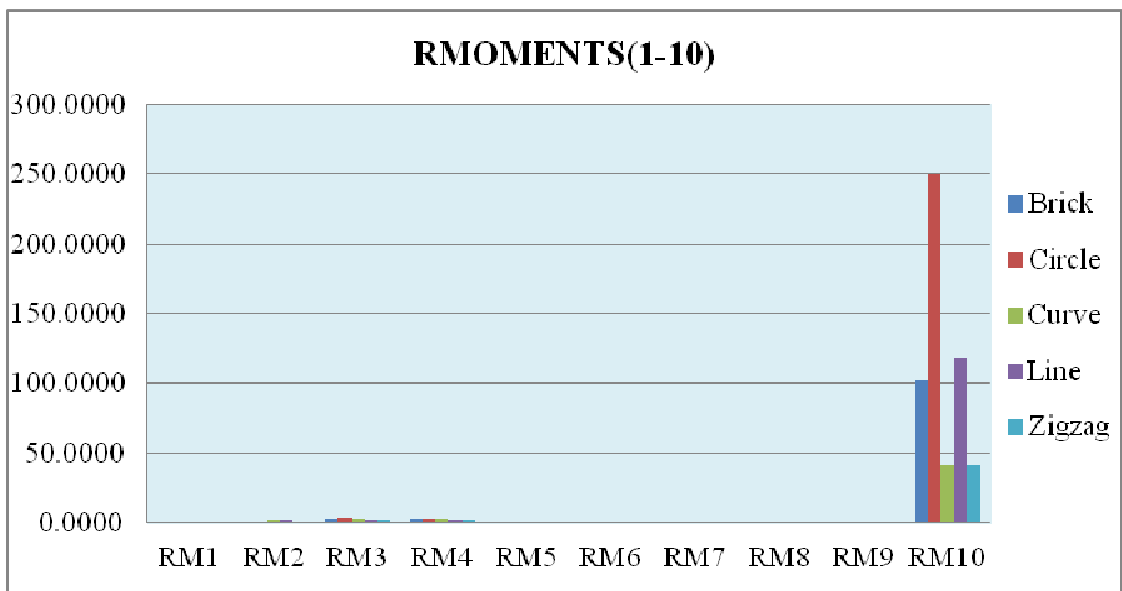

Figure 11. Classification graph on average RM's obtained after preprocessing texture images using local mean

\subsection{Classification on shape texture by RM using local median pre-processing and MS method}

The Algorithm 1 is applied on the same texture images after applying local median preprocessing method. The computed values are given in Table 6. A graph is also displayed based on these values. Figure 12 gives a representation of it.

TABLE 6. AVERAGE RM ON MS SCHEMES OBTAINED AFTER PRE-PROCESSING THE IMAGE USING LOCAL MEDIAN

\begin{tabular}{|l|l|l|l|l|l|l|l|l|l|l|}
\hline Image & RM1 & RM2 & RM3 & RM4 & RM5 & RM6 & RM7 & RM8 & RM9 & RM10 \\
\hline Brick & 0.0830 & 1.0983 & 3.4013 & 2.8371 & 1.1507 & 0.1387 & 0.2040 & 0.1977 & 0.9983 & 90.6251 \\
\hline Circle & 0.0257 & 1.0261 & 3.4835 & 2.6516 & 0.8904 & 0.0722 & 0.1082 & 0.1179 & 0.8420 & 105.3247 \\
\hline Curve & 0.1805 & 1.2119 & 1.2130 & 1.2407 & 1.0669 & 0.3180 & 0.3543 & 0.3416 & 0.8720 & 66.5121 \\
\hline Line & 0.1505 & 1.4473 & 6.3547 & 3.1408 & 1.0918 & 0.2799 & 0.2888 & 0.2452 & 1.0024 & 103.9337 \\
\hline Zigzag & 0.0997 & 1.1100 & 2.3648 & 2.0695 & 1.2327 & 0.2775 & 0.2727 & 0.2137 & 0.8874 & 42.9339 \\
\hline
\end{tabular}

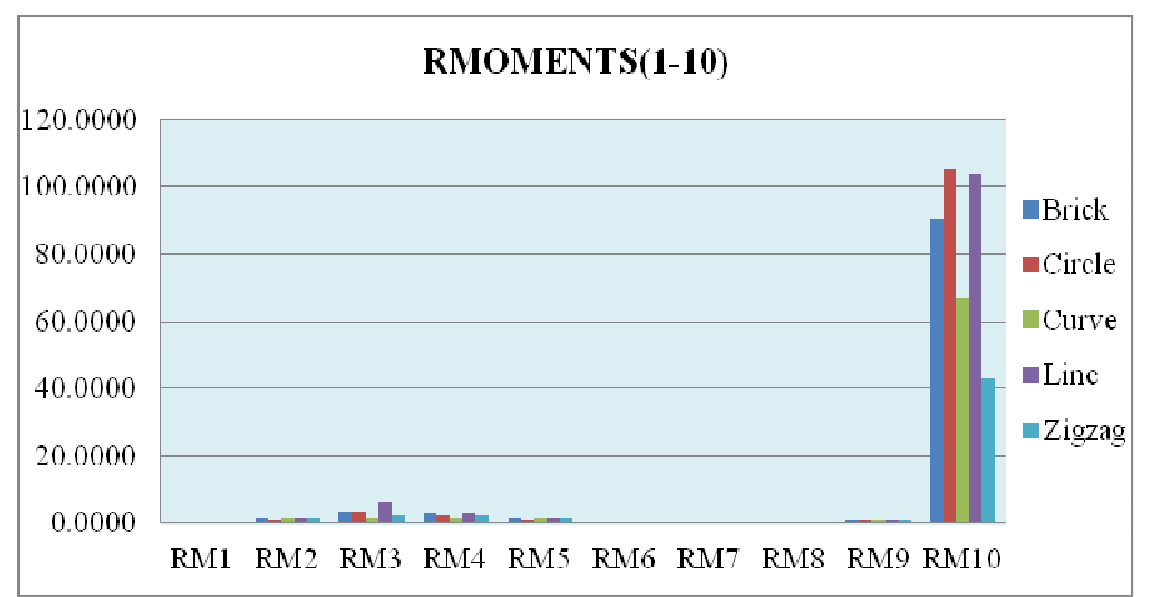

Figure 12. Classification graph on average RM's obtained after preprocessing texture images using local median. 
Signal \& Image Processing : An International Journal (SIPIJ) Vol.4, No.3, June 2013

From this proposed stratagem one can conclude that RM10 alone displays all five groups of textures in a unique manner while other RM's have failed. That is RM1 to RM9 show nearly same values and failed in classification of the textures. The classification obtained after applying local mode preprocessing method is poor when compared to other proposed methods. In all the Circle images are having maximum value than other shape textures after application of these proposed techniques.

\section{Conclusions}

The present paper proposes a novel Morphological Skeleton(MS) representation method on Regional Moments(RM) for classification of textures with similar shape components. A variety of texture classification methods are proposed in the literature for the past three decades. But so far no one has attempted classification of textures based on MS schemes especially using RM. The present paper evaluated RM on MS schemes to classify texture images of different shapes. Classification has been carried out by applying pre-processing methods first followed by extracting the skeleton of the target image on which regional moments are computed to achieve precise classification of textures. The present paper taken into consideration the following preprocessing methods applied on local neighborhoods, which are listed below. a) maximum, minimum, mode, median, mean and maxmin i.e((max-min)/2). Results generated prove that RM10 is sufficient to classify the given group of textures. One need not apply RM1 to RM9 for classification of any texture images as they have totally failed in classification even in the case of pre-processed texture images.

\section{ACKNOWLEDGEMENTS}

The author would like to thank who directly or indirectly helped in carrying out the work. I also thank reviewers for their valuable comments, management of MGIT for their encouragement and family members for their support which led to improvise the presentation quality of this paper.

\section{REFERENCES}

[1] A. Laine \& J. Fan, (1993) "Texture classification by wavelet packet signatures", IEEE Trans. on PAMI, Vol.15, No.11, pp. 1186-1190.

[2] A. Bovik , M. Clark \& W. S. Geisler, (1990)“Multichannel Texture Analysis Using Localized Spatial Filters", IEEE Transactions on Pattern Analysis and Machine Intelligence, Vol.12, No.1, pp. 55-73.

[3] A. K. Jain \& F. Farrokhnia, (1991) "Unsupervised texture segmentation using Gabor filters", Pattern Recognition, Vol.24, No.12, pp. 1167-1186.

[4] M. Unser \& M. Eden, (1989) "Multiresolution feature extraction and selection for texture segmentation", IEEE Transactions on Pattern Analysis and Machine Intelligence, Vol.11, pp. 717728.

[5] Ming-Kuei Hu, (1962) "Visual pattern recognition by moment invariants", Information Theory, IRE Transactions, Vol. 8, pp. 179-187.

[6] Boyce J. F \& Hossack W. J, (1983)"Moment Invariants for Pattern recognition", Pattern Recognition Letters, Vol. 1, pp. 451-456.

[7] Chen C.C. \& Tung-I T Sai, (1993) "Improved moment invariants for shape discrimination", Pattern Recognition, Vol.26, No.5, pp. 683-686.

[8] Sluzek A, (1995) "Identification and inspection of 2-D objects using new moment-based shape descriptors", Pattern Recog., Vol. 16, pp.687-697.

[9] Teaque M. R, (1980)"Image Analysis via the General Theory of Moments", Journal of the Optical Society of America, Vol. 70, pp. 920-930. 
Signal \& Image Processing : An International Journal (SIPIJ) Vol.4, No.3, June 2013

[10] Loncaric S., (1998)“A Survey of Shape Analysis Techniques”, Pattern Recognition, Vol. 31, pp. 9831001.

[11] Lam L., Lee S. \& Suen C., (1992)“Thinning methodologies -A comprehensive survey”, IEEE Transactions on Pattern Analysis and Machine Intelligence, Vol. 14, pp. 869-885.

[12] Smith R.W.,(1987) “Computer Processing of Line Images: A Survey,” Pattern Recognition, Vol. 20, pp. $7-15$.

[13] Chang H.S \& Yan H, (1999)“Analysis of Stroke Structures of Handwritten Chinese Characters", IEEE Trans. Sys. , Vol. 29, pp. 47-61.

[14] Man Cybern B. et al., (1993) "B -spline contour representation and symmetry detection”, IEEE Trans. Pattern Anal. Mach. Intell., Vol. 15, pp. 1191-1197.

[15] Zou J.J and Yan H, (2001) "Skeletonization of Ribbon-Like Shapes Based on Regularity and Singularity Analyses”, IEEE Trans. Sys. Man Cybern. (B), Vol. 31, pp. 391-395.

[16] Hu M. K., (1962) "Visual pattern recognition by moment invariants", IRE 2lan.s. Inform. Theory, IT8, pp. 179-187.

[17] Jia-Guu Leu, (1991) "Computing a shape's moments from its boundary", Pattern recognition, Vol 24, No. 10, pp. 949-957.

[18] Chaur-Chin Chen, (1993) "Improved Moment Invariants for Shape Discrimanation", Pattern Recognition, Vol. 26, No.5, pp.683-686.

[19] H. Sunder \& D. Silver et al, (2003) "Skeleton Based Shape Matching and Retrieval", Proceedings of the shape modeling International, pp. 130-290.

[20] Gang Xu \&Yuqing Lei, (2008) “A New Image Recognition Algorithm Based On Skeleton”, IEEE International Joint Conference on Neural Network, pp. 777-782.

[21] Zhihu Huang \& Jinsong Leng, (2010) “Analysis of Hu’s Moments Invariants on Image Scaling and Rotation”, IEEE $2^{\text {nd }}$ International Conference on Computer Engineering and Technology, Vol.7, pp.476-480.

[22] V. Vijaya Kumar, et al , (2007) "A measure of patterns trends on various types of preprocessed images”, IJCSNS, Vol.7, No.8, p.p. 253-257.

[23] B.V. Ramana Reddy \& A. Suresh et al, (2009)“Classification of Textures based on Features Extracted from Preprocessing Images on Random Windows", International Journal of Advanced Science and Technology, Vol. 9, pp. 9-18.

[24] Andrzej Sluzek, (1995)“Identification and inspection of 2-D objects using new moment-based shape descriptors", Pattern Recognition Letters, Vol. 16, pp. 687-697.

[25] Dr V Venkata Krishna \& M Rama Bai, (2012)" Morphological Boundary Based Shape Representation Schemes on Moment Invariants for Classification of Textures", International Journal of Computer Science and Communication, Vol.-III, No.-I, pp.125-130

[26] Dr M Ashok , J SreeDevi \& Dr M Rama Bai, (2012)”An Approach to Noisy Image Skeletonization using Morphological Methods", International Journal of Scientific \& Engineering Research Vol. 3, Issue 9, pp.1-8.

[27] Dr. V.Vijaya Kumar, A. Srikrishna \& Dr. D.V.L.N Somayajulu, (2008)" An Improved Iterative Morphological Decomposition Approach for Image Skeletonization", International Journal of Graphics, Vision and Image Processing, Vol.8, Issue 1, pp. 47-54.

[28] B. Eswara Reddy \& A. Nagaraja Rao et al, (2007)" Texture Classification by Simple Patterns on Edge Direction Movements", IJCSNS, Vol.7, No.11, pp. 221-225.

[29] Rafael C. Gonzalez \& Richard E. Woods, (2008) Digital Image Processing (Third Edition): Prentice Hall.

[30] J. Serra, Image Analysis and Mathematical Morphology,(1982) Academic Press, London. 
Signal \& Image Processing : An International Journal (SIPIJ) Vol.4, No.3, June 2013

Author

Dr. M. Rama Bai received, her B.E degree from Bharathiar University, Coimbatore(T.N) and her M.Tech (CSE) from College of Engineering, Osmania University, Hyderabad. She received her Ph.D. degree in Computer Science from Jawaharlal Nehru Technological University, Kakinada (JNTUK) in 2012. She served Amrita University, Coimbatore and Sri Hindu College of Engineering, Machilipatnam before joining in MGIT for some period. She then joined as Assistant Professor in the Dept of Computer Science \& Engineering, Mahatma Gandhi Institute of Technology

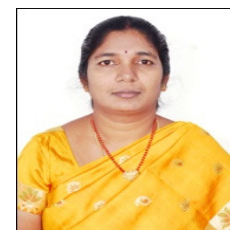
(MGIT) in 1999. At present she is working as Professor in Dept of CSE, MGIT. Her research interests include Image Processing, Pattern Recognition, Digital Water Marking and Image Retrieval Systems. She has published 18 research publications in various National and International Journals and conferences. 